\title{
Enclavement et désenclavement en moyenne montagne d'Europe occidentale - Ardennes belge et française - Morvan - Montagne languedocienne
}

\section{Emmanuel Chiffre}

\section{(2) OpenEdition \\ Journals}

Édition électronique

URL : http://journals.openedition.org/rge/4370

DOI : $10.4000 /$ rge. 4370

ISSN : 2108-6478

Éditeur

Association des géographes de l'Est

Édition imprimée

Date de publication : 1 septembre 1999

ISSN : 0035-3213

Référence électronique

Emmanuel Chiffre, «Enclavement et désenclavement en moyenne montagne d'Europe occidentale Ardennes belge et française - Morvan - Montagne languedocienne », Revue Géographique de l'Est [En ligne], vol. 39 / 4 | 1999, mis en ligne le 02 septembre 2013, consulté le 15 septembre 2020. URL : http://journals.openedition.org/rge/4370

Ce document a été généré automatiquement le 15 septembre 2020

Tous droits réservés 


\title{
Enclavement et désenclavement en moyenne montagne d'Europe occidentale - Ardennes belge et française - Morvan - Montagne languedocienne
}

\author{
Emmanuel Chiffre
}

Thèse de Doctorat de Géographie soutenue en décembre 1997 à l'Université de Limoges, sous la direction du Professeur O. Balabanian, 2 tomes, 603 p., 171 fig., 38 planches photographiques.

1 Ce travail a pour but de présenter les divers aspects caractéristiques et les évolutions d'un milieu géographique original, celui de la moyenne montagne européenne, perçu au travers de trois massifs: Ardenne, Morvan, Montagne languedocienne. La place occupée actuellement par la moyenne montagne dans l'espace d'Europe occidentale, l'intérêt croissant que portent les différents États aux problèmes du renouveau et du développement des espaces montagnards fragiles et la fréquence $\mathrm{du}$ thème du désenclavement dans les différentes réflexions, traduisent bien les préoccupations constantes d'une nécessaire ouverture du milieu montagnard en vue de son intégration à de nouveaux espaces constitués hors des cadres traditionnels, et à ce titre source de nouveaux enjeux.

\section{Caractères originaux de la moyenne montagne et poids des héritages}

2 Les trois massifs retenus comme échantillon, Ardenne, Morvan, Montagne languedocienne, forment un ensemble de régions longtemps comparables, et pourtant inégalement tenues à l'écart des grands courants de circulation et des développements économiques et humains périphériques. Ils s'inscrivent donc dans la recherche de 
formes de désenclavement destinées à rompre un isolement, en général ancien, source de pénalisation et de privation pour les populations locales. Ces trois régions appartiennent à un ensemble d'espaces marginaux situés sur une diagonale NE-SO reliant, du Nord au Sud, Eifel, Massif ardennais, Morvan et Montagne languedocienne, cette dernière correspondant à l'ensemble des hautes terres cristallines entre Tarn, Causses et Montagne Noire, avec les Monts de Lacaune et les Monts de l'Espinouse. Or la particularité de ces espaces tient à la présence d'une périphérie urbanisée et industrialisée; tels le Sillon Sambre et Meuse et l'Ardenne belge ou encore la région industrialisée de Castres-Mazamet proche de la Montagne languedocienne ou bien la zone industrialisée au Sud du Morvan avec Le Creusot-Montceau-les-Mines. Cette association montagne-périphérie impliqua rapidement une série de relations, par exemple le développement de la proto-industrie à partir des XVIe et XVIIe siècles, ou l'existence d'importants foyers de réserve de main-d'œuvre disponible. Mais la similitude des situations tient également à de lourds handicaps : l'altitude, même si elle reste modeste dans bien des cas, la faible taille des communes, sauf en Ardenne belge, depuis la fusion des communes de 1977, ou les faibles densités de population. En 1990, $86,5 \%$ des communes du Morvan et 67,5\% de celles de la Montagne languedocienne ont des densités de population inférieures à $10 \mathrm{hab} . / \mathrm{km} 2$ : ce qui, contrairement à l'Ardenne belge, éloigne les communes d'un pôle attractif, constituant un obstacle à la création des "villages-centres", du style de ceux de l'Ardenne belge. Dans ces conditions, la perception de l'enclavement et de ses effets est jugée parfois comme un facteur contraignant ou bien comme un facteur relatif lorsque la conscience de la proximité du voisin réduit le sentiment d'isolement. Car les conceptions de l'enclavement ont évolué depuis un demi-siècle par suite des changements de mentalités. L'enclavement n'est plus une exclusion, car l'enclavé construit lui-même son propre espace vécu. L'enclavement n'est plus un repli sur soi, ni une absence de contact avec l'extérieur. C'est au contraire une prise de conscience nouvelle dans laquelle l'espace est défini à partir de l'intérieur. Un acte volontariste qui modifie profondément la notion d'espace vécu, source selon les Belges, d'un «nouveau rural », comme dans le cas du pays d'Herve en Ardenne belge. La valorisation personnelle des atouts de la moyenne montagne met fin ainsi à l'exclusion physique et sociale. Le roman de Ferdinand Fabre "Taillevent " fournit d'ailleurs une excellente analyse de l'évolution de l'espace vécu en moyenne montagne languedocienne, à la fin du XIXe siècle, et de l'adaptation des habitants aux contraintes du milieu naturel.

Il existe néanmoins des facteurs contraignants créant l'enclavement dont les divers effets ont pu engendrer une spécificité de la moyenne montagne. Celle-ci se définit plus par ses formes et son articulation que par ses altitudes, situées dans une plage allant de $450 \mathrm{~m}$ (limite de l'étage inférieur du modelé cryogénique atténué) à 1 100-1 $200 \mathrm{~m}$ pour les massifs étudiés. Des massifs à fortes pentes, aux vallées encaissées, isolés, en font un espace accidenté, rude, longtemps considéré comme une montagne obstacle ou une montagne refuge. Même le massif ardennais aux faibles altitudes, n'en présente pas moins certaines caractéristiques évoquées précédemment, permettant de parler de milieu montagnard avec ses contraintes et ses exigences qui doivent être prises en compte par les aménageurs. On mesure les difficiles relations intérieures, l'accès à un pays sévère, à très forte humidité, aux hivers rigoureux et surtout à ruptures climatiques annuelles fréquentes. Un milieu fermé, un relief peu aéré, obstacle à la circulation, donc enclin à l'isolement; ainsi $35 \%$ des communes de la Montagne languedocienne sont situées à une altitude comprise entre 700 et $900 \mathrm{~m}$. En outre, 
altitude et relief combinent leurs effets pour rendre le milieu plus agressif avec de brusques contrastes de températures, de forts gradients pluviométriques, de fortes gelées, du verglas et des chutes de neige très soudains, des brouillards denses et permanents en altitude. Au total, un ensemble de facteurs qui contrarient en hiver la circulation routière, dont les effets sont renforcés par la présence dominatrice de la forêt couvrant en moyenne de $37 \%$ à $42 \%$ du sol. Soit une région d'isolement naturel vouée à la stagnation et au retard dans son développement économique en raison d'un enclavement ancien et tenace : tels se présentaient les Hautes Fagnes, le Morvan et la Montagne languedocienne à la fin du XIXe siècle.

Or sur ces contraintes physiques se greffent parallèlement un ensemble de facteurs renforçant la portée de l'enclavement. Il s'agit des caractères conservateurs de l'ancienne économie rurale qui depuis longtemps ont bloqué toute évolution des structures et renforcé en cela l'enclavement des hautes terres. Les pratiques communautaires anciennes, l'appropriation collective du sol, l'indivision de la terre et les transmissions intégrales, sans partage des biens, ont ainsi favorisé un blocage de toute évolution. Il en résulte la persistance de formes traditionnelles. Ainsi la communauté familiale sous la direction d'un chef de communauté ou maître autoritaire, la communauté de métayers comparables par ses structures, ou encore la mazade, type de concession collective de terres aux habitants, soumis aux mêmes contraintes que la communauté familiale précédente, immobilisent le paysage rural jusqu'à l'époque actuelle comme le montrent les structures de la commune de Fraïssesur-Agout en Montagne languedocienne. Cette fixité du paysage est accompagnée d'un immobilisme des structures et de l'exploitation qui demeure fondamentalement extensive.

Mais l'état de la trame urbaine en moyenne montagne aggrave cette situation déjà bien précaire. Le très faible pourcentage de communes urbaines $(2,5 \%$ à $3,5 \%$ du total des communes) et l'absence de communes urbaines de taille importante pénalisent un développement urbain générateur de relations sur le plan régional. En l'absence de hiérarchies urbaines intermédiaires organisant un espace régional, et avec un réseau urbain périphérique très insuffisant et mal intégré à l'espace régional, la moyenne montagne ne possède pas les conditions favorables à son développement. Tout concourt à un repli et à un effacement de la moyenne montagne qui, de 1920 à 1990, a perdu $80 \%$ de sa population pour plus de la moitié des cantons du Morvan et de la Montagne languedocienne, où les densités sont très faibles, à l'exception de l'Ardenne belge. Il en résulte une faiblesse des équipements et des flux de marchandises et de personnes à l'intérieur des massifs. Une situation qui au total se caractérise par un effacement économique et humain ainsi qu'un renforcement de l'enclavement comme le montrent les valeurs des différents indices des enquêtes INSEE (1988) ou SEGESA (1993). Ces indices, qui définissent pour l'INSEE une moyenne des distances d'accès à une série d'équipements et pour la SEGESA la distance moyenne à des services et des équipements classés en deux catégories selon l'importance et la fréquentation de leur utilisation, sont compris entre $7,6 \mathrm{~km}$ et $11,8 \mathrm{~km}$ pour le Morvan et la Montagne languedocienne, caractérisant un fort enclavement contrairement à l'Ardenne belge. Ce que confirme les éloignements de la gare, de l'autoroute ou de la desserte en autocar, facteurs décisifs de l'enclavement (tableaux 1 et 2). 
Tableau 1 : Enclavement et distances aux gares de voyageurs - lignes d'autocar - stations de taxi en Morvan

\begin{tabular}{|c|c|c|c|c|c|c|c|c|}
\hline \multirow{2}{*}{$\begin{array}{c}\text { Paramètres mesurant } \\
\text { I'enclavement } \\
\text { Cantons } \\
\text { du Morvan }\end{array}$} & \multicolumn{2}{|c|}{ Indice d'enclavement } & \multicolumn{2}{|c|}{ Gares de voyageurs } & \multicolumn{2}{|c|}{ Lignes d'autocar } & \multicolumn{2}{|c|}{ Stations de taxi } \\
\hline & $\begin{array}{c}\text { Indice } \\
\text { SEGESA }\end{array}$ & $\begin{array}{c}\text { Evolution } \\
80-88\end{array}$ & $\begin{array}{l}\text { Nbre de } \\
\text { communes }\end{array}$ & $\begin{array}{l}\text { Distance } \\
\text { en km }\end{array}$ & $\begin{array}{l}\text { Nbre de } \\
\text { communes }\end{array}$ & $\begin{array}{c}\text { Distance } \\
\text { en km }\end{array}$ & $\begin{array}{l}\text { Nbre de } \\
\text { communes }\end{array}$ & $\begin{array}{l}\text { Distance } \\
\text { en } \mathrm{km}\end{array}$ \\
\hline Château - Chinon & 10,2 & + & 0 & 62 & 6 & 3 & 4 & 9 \\
\hline Corbigny & 11,9 & + & 0 & 12 & 7 & 7 & 0 & 12 \\
\hline Fours & 9 & + & 1 & 11 & 1 & 11 & 1 & 12 \\
\hline Lormes & 10,2 & + & 0 & 21 & 2 & 10 & 4 & 13 \\
\hline Luzy & 7,5 & + & 4 & 8 & 1 & 0 & 4 & 7 \\
\hline Montsauche-les-Settons & 10,3 & + & 0 & 34 & 0 & 20 & 5 & 7 \\
\hline Moulins - Engilbert & 8,6 & + & 0 & 20 & 4 & 11 & 3 & 7 \\
\hline Quarré - les - Tombes & 9,8 & + & 0 & 16 & 0 & 10 & 2 & 14 \\
\hline Lucenay - l'Evêque & 8,9 & - & 2 & 17 & 8 & 0 & 2 & 13 \\
\hline St - Léger / Beuvray & 7,4 & - & 2 & 12 & 4 & 7 & 2 & 11 \\
\hline Liernais & 10,8 & - & 3 & 27 & 0 & 13 & 1 & 13 \\
\hline Saulieu & 8,2 & + & 5 & 15 & 2 & 11 & 3 & 8 \\
\hline TOTAL & 9,3 & - & 17 & 21 & 35 & 9 & 31 & 10 \\
\hline
\end{tabular}

Tableau 2 : Enclavement et distances aux gares de voyageurs - lignes d'autocar - stations de taxi en Montagne languedocienne

\begin{tabular}{|c|c|c|c|c|c|c|c|c|}
\hline \multirow{2}{*}{$\begin{array}{c}\begin{array}{c}\text { Paramètres mesurant } \\
\text { l'enclavement }\end{array} \\
\begin{array}{c}\text { Cantons de la Montagne } \\
\text { languedocienne }\end{array}\end{array}$} & \multicolumn{2}{|c|}{ Indice d'enclavement } & \multicolumn{2}{|c|}{ Gares de voyageurs } & \multicolumn{2}{|c|}{ Lignes d'autocar } & \multicolumn{2}{|c|}{ Stations de taxi } \\
\hline & $\begin{array}{c}\text { Indice } \\
\text { SEGESA }\end{array}$ & $\begin{array}{c}\text { Evolution } \\
80-88\end{array}$ & $\begin{array}{l}\text { Nbre de } \\
\text { communes }\end{array}$ & $\begin{array}{l}\text { Distance } \\
\text { en km }\end{array}$ & $\begin{array}{l}\text { Nbre de } \\
\text { communes }\end{array}$ & $\begin{array}{l}\text { Distance } \\
\text { en km }\end{array}$ & $\begin{array}{l}\text { Nbre de } \\
\text { communes }\end{array}$ & $\begin{array}{c}\text { Distance } \\
\text { en km }\end{array}$ \\
\hline Alban & 9 & + & 0 & 32 & 3 & 12 & 1 & 11 \\
\hline Anglès & 13,6 & + & 0 & 35 & 0 & 32 & 0 & 15 \\
\hline Brassac & 8,6 & + & 0 & 25 & 2 & 5 & 1 & 8 \\
\hline Dourgne & 7,4 & + & 0 & 24 & 8 & 6 & 0 & 10 \\
\hline Lacaune & 10,8 & - & 0 & 46 & 3 & 10 & 1 & 13 \\
\hline Montredon-Labessonnié & 13 & + & 0 & 26 & 1 & 20 & 0 & 17 \\
\hline Murat-sur-Vèbre & 12 & - & 0 & 65 & 2 & 6 & 4 & 13 \\
\hline Roquecourbe & 4,3 & + & 0 & 16 & 3 & 7 & 2 & 9 \\
\hline St-Amans-Soult & 5,7 & + & 0 & 16 & 7 & 4 & 1 & 9 \\
\hline Vabre & 13,9 & + & 0 & 37 & 3 & 12 & 2 & 15 \\
\hline Belmont-sur-Rance & 9,6 & - & 0 & 38 & 2 & 11 & 1 & 13 \\
\hline Camarès & 13 & + & 0 & 42 & 3 & 15 & 1 & 14 \\
\hline St-Sernin-sur-Rance & 11,4 & - & 0 & 48 & 3 & 15 & 3 & 10 \\
\hline Mas-Cabardès & 14,8 & + & 0 & 23 & 6 & 8 & 1 & 25 \\
\hline Saissac & 10,9 & + & 0 & 25 & 5 & 4 & 0 & 24 \\
\hline Bédarieux & 7 & - & 2 & 12 & 8 & 5 & 2 & 7 \\
\hline Lunas & 11,7 & + & 4 & 23 & 2 & 10 & 1 & 13 \\
\hline Olargues & 10,8 & + & 0 & 30 & 10 & 0 & 2 & 9 \\
\hline St-Gervais-sur-Mare & 6,9 & - & 0 & 11 & 7 & 4 & 3 & 5 \\
\hline St-Pons-de-Thomières & 12,9 & + & 0 & 51 & 4 & 9 & 1 & 13 \\
\hline La Salvetat-sur-Agout & 12,7 & - & 0 & 68 & 2 & 10 & 0 & 19 \\
\hline TOTAL & 10,6 & - & 6 & 33 & 84 & 10 & 27 & 13 \\
\hline
\end{tabular}

\section{Les stratégies d'ouverture de la moyenne montagne lors du premier désenclavement au XIXe siècle}

6 Une conjonction de forces dynamiques et le développement du chemin de fer à la fin du XIXe siècle constituent les bases du premier désenclavement contrarié par les résistances et phases d'inertie qui limiteront la portée de l'ouverture, puis la construction d'un nouvel espace de communications. Route et train offrent en effet la possibilité de reconstruire un espace économique et démographique plus cohérent, 
prenant appui sur une meilleure desserte. Mais la révolution industrielle liée à la révolution des transports impose des choix, des stratégies qui ne sont pas toujours en conformité avec les exigences de la moyenne montagne. Les notions de rentabilité, d'utilité des nouveaux réseaux de communications, la desserte à différents degrés détournent le désenclavement de sa fonction humaine.

7 La difficile adaptation du réseau routier à la moyenne montagne résume ces réserves. Le développement routier de la première moitié du XIXe siècle se heurte à l'obstacle du relief, au repli économique et humain, à une grande dispersion de l'habitat, à la carence des anciens chemins. Or la route impose sa logique administrative, économique, humaine, à l'image des routes du sel, ou des routes royales du XVIIIe siècle, face à une circulation difficile, souvent précaire. L'ancien réseau archaïque, insuffisant, est inutilisable une partie de l'année : fin XVIIIe siècle, 44 \% des routes du Morvan sont en très mauvais état, $23 \%$ en Montagne languedocienne. Un réseau au total insuffisant, mal adapté aux besoins. Tel est le cas de la Haute Ardenne belge où les Hautes Fagnes sont inaccessibles une partie de l'année, ou encore du Morvan, jugé inabordable à la fin du XVIIIe siècle par l'ingénieur Dumorey, dont $60 \%$ à $75 \%$ des routes ne sont pas praticables en hiver. L'ouverture passe donc par un réseau moderne de routes, complétant et consolidant le réseau routier antérieur seul apte à rattacher les espaces montagnards isolés au maillage des grandes routes en cette première moitié du XIXe siècle.

8 Le développement industriel à la périphérie de certains massifs (Sud de la Montagne languedocienne), un vif esprit d'entreprise, la volonté de renouveau et de concentration dans le secteur industriel vont favoriser l'implantation du chemin de fer, source d'une accélération de la circulation puis de l'essor des échanges et de la chute des coûts de transports. Mais en dépit des réalisations, les inégalités du désenclavement et $\mathrm{du}$ développement subsistent, comme par exemple entre une Ardenne belge dynamique et un Morvan passif. Les effets du rail sont néanmoins positifs. Le chemin de fer entraîne une transformation profonde de la vision traditionnelle du monde extérieur par l'introduction du mouvement, de la vitesse et d'une dimension nouvelle dans les rapports humains, ce dont profite la montagne. Le chemin de fer est réclamé par les populations rurales tout au long de la deuxième moitié du XIXe siècle, car il est le symbole de la valorisation des produits locaux, de l'accès à de nouveaux marchés. Symbole de progrès, il apparaît, avec la gare, comme le moteur de la rupture de l'isolement rural mais en même temps comme le triomphe de la civilisation industrielle et de la ville sur la moyenne montagne. Cette idée chère aux Saint-Simoniens, devient dès 1829 un thème favori de réflexion politique. Le tracé de grands axes, la desserte des régions périphériques et des régions de montagne (Plan Freycinet, ouverture de l'Ardenne belge vers le Rhin, ouverture du Massif Central vers Paris et le Nord de la France dans la deuxième moitié du XIXe siècle) en sont l'illustration. Mais parallèlement aux lignes principales apparaît une génération de réseaux ferrés d'intérêt local et régional, les « Petits Trains » ou les « Tacots ». Cette réalité repose sur le rôle de quelques grandes compagnies (Compagnie du Midi, Compagnie du PLM) qui, à partir de 1852, se partagent l'espace de la moyenne montagne. L'élan est ainsi donné pour un nouvel espace national. Les réalisations ferroviaires qui en découlent sont multiples et diverses dans leurs effets : désenclavement de l'Ardenne belge et essor des voies ferrées avec les lois de 1834 et 1837, création de la Société Nationale des Chemins de Fer en 1885, mise en place du réseau de chemins de fer vicinaux. Grâce au rail, 
l'Ardenne belge devient un trait d'union en Europe de l'Ouest. Le désenclavement touche aussi le Morvan avec les "Tacots " et "Petits Trains" qui, de 1880 à 1940, revitalisent les espaces isolés, comme l'illustre l'exemple de la ligne Nevers-Saulieu de 1901 à 1940. De même le désenclavement de la Montagne languedocienne s'effectue par des tracés de lignes minières (Béziers-Graissessac), de lignes intramontagnardes (Béziers-Neussargues), de lignes de piémont (Bédarieux-Castres). Trois types d'interventions qui se complètent par la création des «Petits Trains tarnais » (CastresLacaune-Murat-sur-Vèbre). Le rail joue ainsi un rôle fondamental dans le développement, d'autant plus que l'association rail-route-voie d'eau renforce la pénétration de la moyenne montagne et l'emprise de l'extérieur sur les différents massifs.

9 Une autre forme de désenclavement s'affirme avec le passage de la culture populaire à la culture nationale. Ce désenclavement des esprits, consécutif aux désenclavements routier et ferroviaire, marque également le triomphe des nouveaux moyens de communication qui imposent de penser, de concevoir, d'organiser différemment. Au cadre familial étroit, aux mentalités traditionnelles, se substituent la circulation des idées, du savoir, puis l'émergence, au lieu et place de la langue ancienne, d'une langue nationale, symbole de culture urbaine. Une volonté de changement s'affirme dans les esprits : les patois, langues parlées des générations passées, langues de la tradition, témoins d'un certain archaïsme des structures rurales s'effacent. Ainsi, en Montagne languedocienne, $84 \%$ des anciennes générations âgées de plus de 70 ans conservent encore en 1992 la pratique de l'occitan contre $8 \%$ seulement pour les jeunes générations âgées de moins de 25 ans. Le désenclavement des esprits ouvre à la vie moderne l'espace jusque-là confiné et statique de la moyenne montagne. En même temps qu'il impose une nouvelle gestion de cet espace, il crée de nouvelles solidarités. C'est la conquête de la moyenne montagne par une culture nationale qui s'impose aux différentes générations. Mais également la prise de conscience de l'émergence d'une certaine unité au niveau de l'État par l'emprise des réseaux de communication sur l'espace géographique. De plus la moyenne montagne languedocienne s'était antérieurement identifiée à cette évolution par l'effacement du catharisme et l'intégration du mouvement protestant, avec l'affaissement de l'idée de montagnerefuge.

\section{La poursuite récente et le renouveau du désenclavement en moyenne montagne}

10 La moyenne montagne doit réagir pour affirmer sa place dans l'espace rural, pour valoriser ses atouts, pour s'intégrer aux nouveaux schémas de relations à grande distance qui se tissent au niveau européen. Mais le peut-elle encore? De fréquentation plus faible que la haute montagne, d'attraction modeste, de conditions d'altitudes et de climat médiocres, qui limitent une utilisation permanente en toutes saisons, il faut réfléchir à ses options futures. Par exemple développer le tourisme vert orienté vers la recherche du patrimoine, des traditions, ou plutôt recomposer le paysage en prenant appui sur les structures des parcs naturels et des réserves. Ce qui implique un tissu démographique rénové, une forte mobilité de la population active par l'effet "navette", un comportement social renouvelé. Car vivre en moyenne montagne suppose un choix, un mode de vie différent et de nouvelles solidarités : c'est-à-dire 
repenser tout le cadre de vie antérieur. Mais parallèlement on ne peut ignorer l'évolution de la moyenne montagne qui modifie les réactions face à l'enclavement. Jadis espace de vie quotidien, celle-ci est souvent devenue un espace de loisirs, ce qui confère plus de relativité à la notion d'enclavement. Milieu fragile, zone de revitalisation rurale depuis 1995, à faibles densités (de 8 à 15 hab./km2 en Montagne languedocienne), à recul important de population active (plus de $10 \%$ de 1982 à 1990 en Morvan), tout concourt au déclin de la moyenne montagne, comme le confirme l'importance des migrations définitives et des résidences secondaires. C'est pourquoi les parcs naturels, par leur vocation de conservation du milieu associée à la gestion de l'espace, peuvent ralentir un déclin qui apparaît inévitable. Tels sont les exemples du Parc Naturel des Hautes Fagnes-Eifel en Ardenne, du Parc Naturel du Haut-Languedoc ou de celui du Morvan. De même les « autoroutes de l'information » peuvent apporter un soutien efficace aux tentatives de renouveau rural.

11 Dans cette entreprise, la multiplication de moyens modernes de communication (autoroutes, TGV) peut constituer le support du développement. L'autoroute assure de nouvelles fonctions, à un niveau supérieur, dans un espace plus vaste qui associe la moyenne montagne à de nouveaux espaces de relations. Vecteur et outil du développement, l'autoroute représente un moyen nouveau de communication et surtout de diffusion. Mais les problèmes d'accessibilité et de desserte, les coûts d'investissement associés aux coûts d'exploitation, la nécessaire rentabilité du projet, posent la question de l'implantation des formes modernes d'accès à la moyenne montagne et à sa marginalité. Il y a là un enjeu dans cette lutte contre le déclin et la désertification des régions les plus isolées, comme le prévoit le Schéma Directeur Routier National de 2015. Les stratégies retenues seront donc adaptées aux conditions particulières et aux contraintes de chaque milieu spécifique, à partir d'une réflexion sur le rôle et l'utilité des moyens par rapport aux exigences du milieu de moyenne montagne qu'il convient d'irriguer. Mais jusqu'où et à quel prix? Des solutions proposées montrent les limites de l'aménagement de l'espace. Elles tentent de résoudre l'enclavement de vastes espaces et leur rattachement à de grands axes de relations (figures 1,2 et 3). Si le modèle de l'Ardenne belge peut être retenu comme un succès certain du désenclavement, si le Morvan, obstacle ancien et traditionnel, reste pénalisé par l'échec des tentatives de relations Est-Ouest, seule la Montagne languedocienne a tenté de combler son retard à partir de la mise en place de trois axes de relations à grande distance. Tel l'axe européen Toulouse-Lyon, en cours d'aménagement à partir de la N88, ou encore l'autoroute A75 (Clermont-Ferrand - Béziers) qui traverse le Massif Central dans sa partie médiane, ou enfin la N112 d'Albi à Béziers. Ils constituent des projets très ambitieux, inégaux dans leurs réalisations, facteurs d'un futur développement régional et d'une rénovation économique. Mais les résultats de cette ouverture de l'espace rural restent actuellement modestes. Ainsi l'autoroute A75, autoroute d'État gratuite, aux sorties rapprochées, dessert linéairement le Massif Central en insistant plus sur la fonction de passage que sur celle d'aménagement. La desserte l'emporte sur le développement spatial et sur la rupture des espaces les plus isolés. Seule une expérience de plusieurs années permettra d'approfondir les conséquences humaines, économiques et spatiales. Mais en même temps l'enclavement n'est pas nécessairement négatif, selon qu'il est vécu de l'intérieur ou considéré de l'extérieur de la moyenne montagne. Au total, autant de réflexions, d'essais, d'originalité dans la recherche d'une efficacité économique et d'un renouvellement des différentes potentialités. 
Fig. 1 : Le réseau routier actuel de l'Ardenne belge (1995)

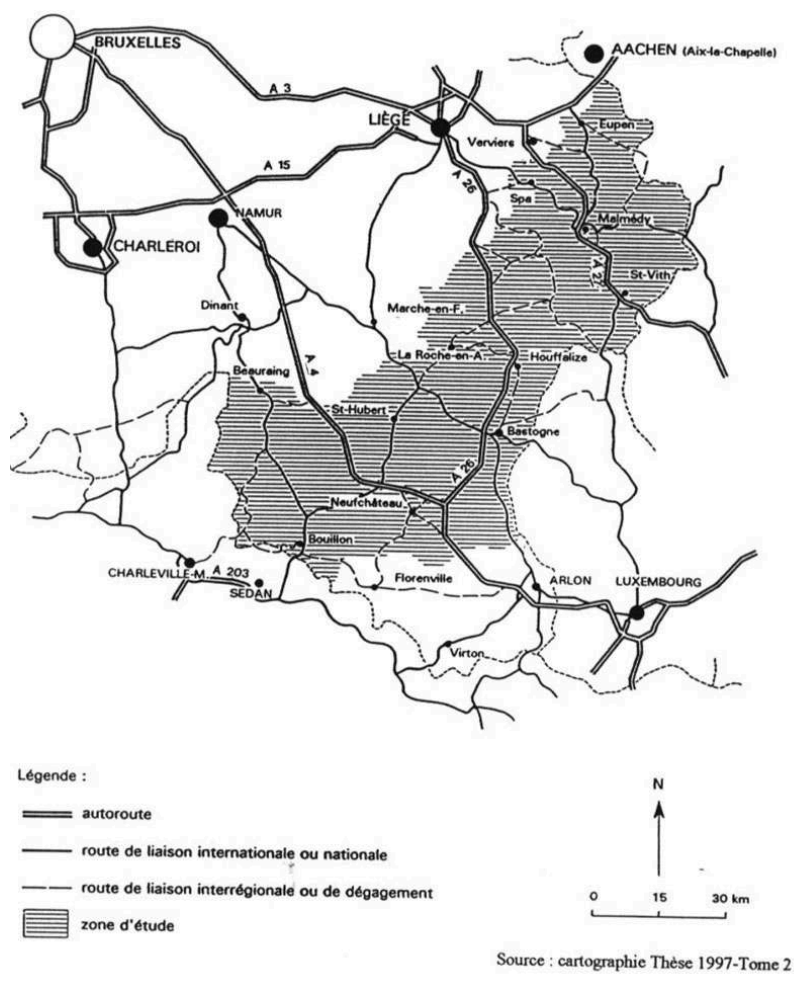

Fig. 2 : Trafic routier dans le Morvan (trafic journalier moyen annuel) (1992)

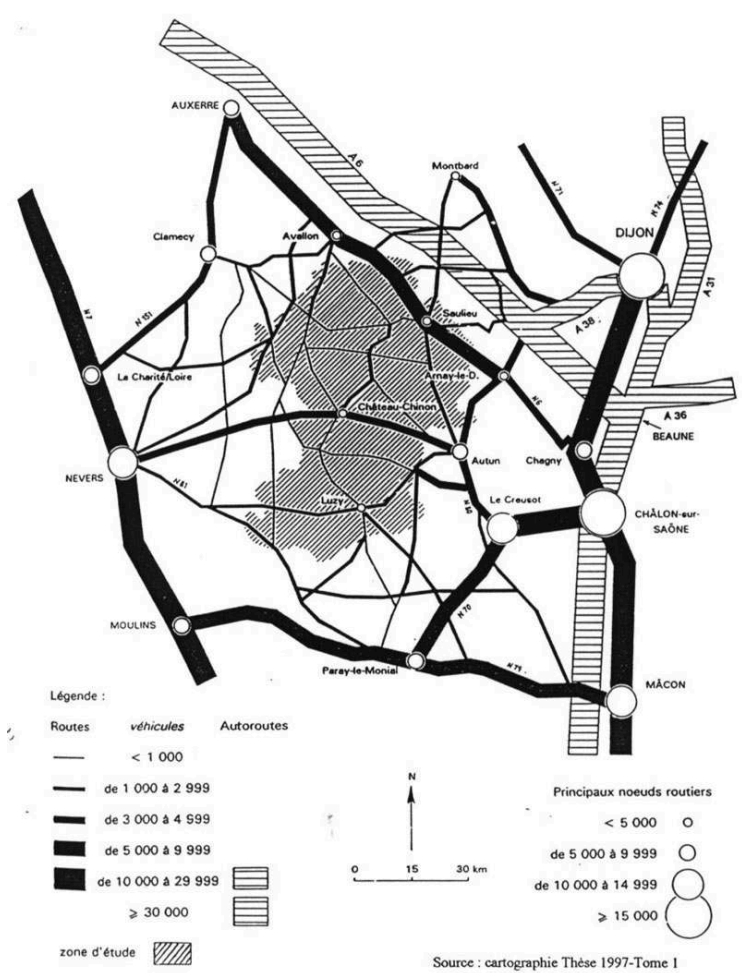


Fig. 3 : Trafic routier en Montagne languedocienne (trafic journalier moyen annuel) (1992)

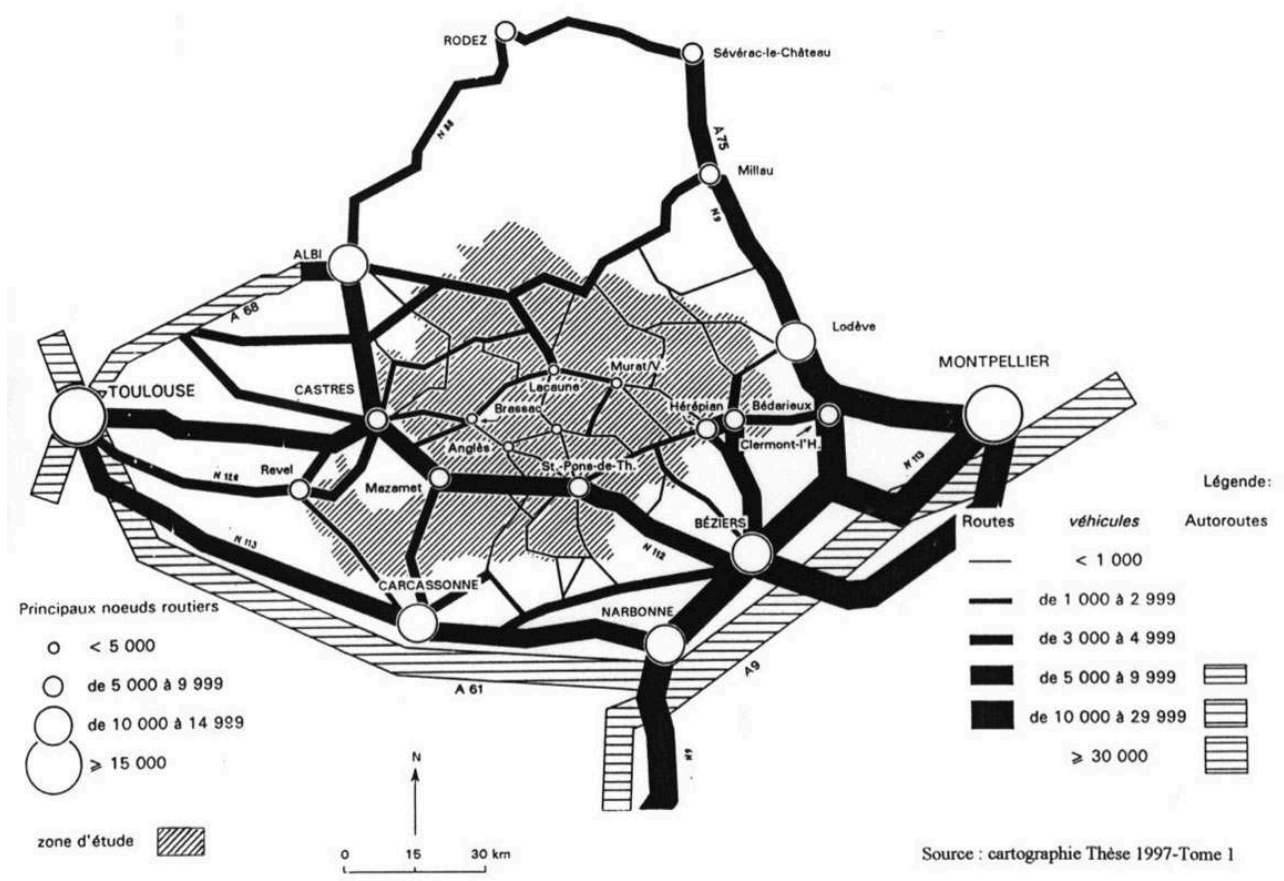

Ces réflexions impliquent une comparaison à l'échelle européenne des milieux voisins de moyenne montagne qui, confrontés à des situations et des problèmes identiques, en ont tiré des thèmes de réflexion puis des comportements particuliers. Pour ces raisons, en prolongement de l'étude Ardenne-Morvan-Montagne languedocienne, une recherche sur le Mittelgebirge allemand apporterait une contribution à une réflexion d'ensemble. Car la moyenne montagne allemande, à l'image de certains massifs, à forte identité, très caractéristiques (Harz - Thüringer Wald - Erzgebirge - Forêt Noire - Eifel), a su s'adapter tout au long de son histoire aux évolutions démographiques, économiques et sociales. Elle a adopté une démarche qui passe par un enrichissement des possibilités de diversification vers de nouvelles formes industrielles de haute technologie et de spécialisation ; mais également vers une option du développement du tourisme de double saison (hiver-été) et de double accueil (thermal - loisirs) qui, par exemple, place le Harz et la Forêt Noire en position privilégiée pour le contrôle du potentiel du ski allemand. Enfin le rétablissement en 1990 de l'unité du territoire allemand encourage et amplifie la recherche de nouvelles formes de développement dans les milieux de moyenne montagne. Mais les solutions proposées sont-elles applicables aux autres massifs?

En conclusion, l'analyse de la moyenne montagne fait apparaître à la fois une très grande diversité des espaces la composant, en même temps qu'une impression d'ensemble d'unité du milieu. Il existe bien un milieu original et spécifique de moyenne montagne où l'on retrouve les grands lignes d'identification de la montagne: tels le phénomène de réservoir de main-d'œuvre durant la deuxième moitié du XIXe siècle, ou encore l'importance de la proto-industrie dans le développement économique, ou enfin l'organisation d'un milieu économique et humain longtemps en symbiose avec l'avantpays. La moyenne montagne peut-elle retrouver son ancienne influence? Quel peut être son avenir? Toutes ces questions se posent inévitablement lorsque l'on considère l'évolution actuelle des différentes régions de moyenne montagne. Certes elles ne sont 
plus, de nos jours infranchissables, les moyens modernes de communication pouvant contribuer à leur développement futur. Mais ces régions fidèles à un passé, très attachées à leur ancien développement, ne peuvent rester insensibles ni passives face à l'évolution actuelle qui les conduit à une situation d'«arrière-pays ». En fondant leur démarche future sur la relativité de l'enclavement, elles peuvent avoir une vision plus réaliste des enjeux et des choix et éviter ainsi de devenir progressivement des espaces marginalisés.

\section{AUTEUR}

\section{EMMANUEL CHIFFRE}

Maître de Conférences - Département de géographie - Université de Nancy 2, BP 339754015

Nancy Cedex 